\title{
Social Media as a Tool to Look for People with Dementia Who Become Lost: Factors That Matter
}

\author{
Kelvin KF Tsoi ${ }^{1,2}$, Lingling Zhang ${ }^{1}$, Nicholas B Chan ${ }^{1}$, Felix CH Chan ${ }^{1}$, Hoyee W Hirai ${ }^{1}$, Helen ML Meng ${ }^{1,3}$ \\ ${ }^{1}$ Stanley Ho Big Data Decision Analytics Research Centre \\ ${ }^{2}$ Jockey Club School of Public Health and Primary Care \\ ${ }^{3}$ Department of Systems Engineering and Engineering Management \\ The Chinese University of Hong Kong \\ kelvintsoi@cuhk.edu.hk
}

\begin{abstract}
This research explored how social media were used to look for people with dementia who went lost, and investigated what features of social media usage were associated with the outcomes of finding. Tweets that were disseminated to find missing people with dementia were collected and clustered by cases. Ten cases were selected as sample cases and traced for the outcomes of finding. Information of the Twitter users who tweeted and retweeted were retrieved and categorized. Descriptive analysis was applied to examine the lost cases and features of social media usage; T-test and chi-square analysis were conducted between outcomes of the lost incidents and key features of tweets and Twitter users. Results indicated that there was no significant association between the average number of tweets and retweets and the outcomes of finding, but social media users, especially the ones with a larger group of followers (audience), such as the media, should be encouraged to spread such information. However, a code of conduct is needed to ensure social media are not abused.
\end{abstract}

\section{Introduction}

Dementia is a public health priority recognized by the World Health Organization [1]. It is a syndrome of a complex, irreversible and relentlessly progressive loss of cognitive function [2]. The number of people with dementia was estimated at 47 million worldwide in 2015 [3] and as the population ages, the number of persons with dementia continues to rise [4]. Increasing at a speed of one new case per three seconds, each year nearly 9.9 million new cases emerge and there would be 132 million people with dementia around the world by 2050 [3]. A variety of brain illnesses can cause dementia, among them Alzheimer's disease (AD) is the most common one, contributing to $60 \%-70 \%$ of cases $[1,5]$. $\mathrm{AD}$ is a progressive deterioration of cognitive and functional impairment [5]. The cause of $\mathrm{AD}$ is poorly understood and there is no cure [6]. The foremost known risk factor of dementia is age. In the general population aged 60 and over, about five to eight in every 100 people are estimated to have dementia [1]. Dementia is the major causes of dependence and subsequent care among senior people [7]. However, there has also been an increasing awareness of the impact of dementia on people at a younger age [8]. Younger onset dementia (dementia diagnosed in people before the age of 65) accounts for up to $9 \%$ of cases [1].

The risk of people with dementia getting lost is substantial [9]. Previous research suggested that getting lost is associated with "wandering" [10], a term which has different definitions in different studies, but most of them include moving oneself through space and being cognitively impaired [11]. However, later research suggested that wandering and missing incidents are two different behaviors [12-15] and that there is little or no evidence to suggest that persons with dementia who wander will be more likely to become lost [16].

This research focused on the information disseminated on social media in an attempt to look for people with dementia who got lost, defined as they were away from their expected location and unable to return independently, no matter how long or where they were lost. We studied some key features of social media usage and investigated whether of the features were associated with the outcomes of the finding efforts. The research did not study whether the usage of social media would facilitate the finding outcomes. 
Tweets that contain terms of both "dementia" or "Alzheimer" as well as "lost" or "missing" were collected for a period of two months, namely, AprilMay, 2017 through Twitter REST application programming interfaces (APIs) [17]. Natural language processing and manual coding were applied to cluster the tweets into cases. Ten cases from the first experimental month were selected and traced for 30 days. Their demographics were extracted from tweets or webpages linked to through the URLs contained in the tweets. The outcomes of the finding efforts were traced. Information of Twitter users who tweeted and retweeted were also collected through Twitter API and these Twitter users were categorized into three types: media, police and other. Descriptive analysis was conducted to examine the demographics and key features of social media usage. Associations between the features of the tweets and Twitter users and the outcomes of the finding efforts were investigated by $\mathrm{T}$-test and chi-square analysis.

Results showed that there was no significant correlation between the average number of tweets and retweets and the outcomes of finding, yet the involvement of social media users with a larger number of followers (which means they have a large audience) such as the media associated significantly with the outcomes of finding. However, a code of conduct needs to be formed among social media users when spreading such information that look for the lost persons with dementia so as to ensure the information circulating on the social media is correct, intact and updated in time to make the best use of social media and to avoid a waste of public resources.

\section{Background}

Getting lost is a common and unpredictable phenomenon among people with dementia. McShane et al. found in a five-year longitudinal study of 104 community-dwelling people with dementia that over that $66 \%$ of the participants demonstrated apparent risk of getting lost, over $41 \%$ of them got lost at least once and 5 of them repeatedly got lost [9]. Bowen et al. in a study of veterans with dementia for 12months found the risk for missing incident was $46 \%$ [13]; Hwang et al. found $71 \%$ of AD sufferers got lost [18]; Holzer and Warshaw found that 30\% of early AD patients got lost while driving [19]. Research showed that people with dementia would become lost while performing everyday activities that they normally completed without incident [4, 13]. When getting lost, the majority of people with dementia could not safely find their way back to a safe location, due to they have difficulties processing information and are unable to take appropriate actions such as seeking help, responding to searchers or displaying effective problem-solving strategies [4, $13,20]$. Therefore, safety of is an overriding concerning for the persons' family members and caregivers [21].

If people with dementia get lost, they can be in danger. Previous research found that people who became lost can suffer from injuries, and some of them were even found dead [2, 4, 19]. Hunt et al. conducted a study using newspaper and television reports over 10 years (1998-2008) on people with dementia who became lost while driving. Their results showed that among the 207 people with dementia who were lost while driving, $32(15 \%)$ of them were found dead and another 35 (17\%) were found injured [2].

If people with dementia are lost, it is critical that they are located within 24 hours. Bowen et al. found that in most of the lost cases, the missing persons were found within five to ten minutes $(43.6 \%)$ and most of the missing persons were found near the place they were last seen (61.4\%) [13]. However, the lost persons could also be miles away (12.8\%) [13], and those who are who are missing for longer time are more likely to sustain significant harm $[15,20]$. Koester and Stooksbury found from 42 cases from search and rescue records that there was a $19 \%$ mortality rate, and if the person was not found within 24 hours, the mortality would rise to $46 \%$ [20]. For people with dementia who are missing on foot longer than 24 hours, $50 \%$ might experience a serious injury or die [14], and for people with dementia who are missing while driving and not found within 24 hours, there is a high likelihood of dying from exposure or other occurrence [2,4]. As a result, people with dementia who have experience of becoming lost are more vulnerable of being locked at home or institutionalized $[9,13]$ as caregivers would become stressful $[10,23]$ and $44.8 \%$ of the caregivers worry the persons would get lost in future [24].

Due to the dangerous consequences of missing incidents, it is important that the family members and caregivers look for helps from neighbors, friends, police and the public through effective medium for the lost persons' quick and safe return. Research have proven the importance of persons other than the caregivers in locating the missing people with dementia. Bowen et al. indicated that in 59\% of the cases, the lost persons were found by someone other than caregivers, but neighbours, store employees and Good Samaritans [13]. Rowe's study also indicated that most lost people were found by police officers (36\%) and Good Samaritans (34\%) instead of the caregivers [25]. A delay in a missing person report 
being made would increase the length of time that the persons remain missing [15]. In some places, initiatives have been launched to mobilize social resources in finding the lost persons. Among them are the "Silver Alert" programs established in many states of the United States. When activated, the alerts would provide information to parties including law enforcement agencies such as the department of transportation and media outlets so that the alerts could be broadcast on highway messaging signs and the media [26]. These alert programs have been proved as effective [22], but research found there is still space to mobilize the public in locating the lost persons. A research on missing drivers with dementia found that such alerts were more likely to lead to the recovery of the lost by law enforcement officials (21.4\%) rather than Good Samaritans (2.3\%) [22]. Similarly in the United Kingdom, a research using four-year missing-person record found that 119 $(42.3 \%)$ out of the total among 281 cases were found by police, 59 were found by a family member and someone know the missing person and 50 by a member of public [15].

With the emergence of social media as one of the largest and fastest information sources, people are also using social media to look for missing people with dementia. The popularity of social media, the interactive Web 2.0 Internet-based applications whose content are user-generated [27], has been everincreasing worldwide. The number of social media users broke through 2 billion in 2015 and is expected to reach nearly 3 billion by 2020 [28]. As of 2016, more than $68 \%$ of internet users were social media users [28]. Twitter, a platform which allows users to post short messages, i.e., tweets, of no longer than 140 characters, is one of the most popular social media. It has 1 billion unique visits monthly to sites and 313 million monthly active users [29]. Through Twitter APIs, large scales of Twitter data, including tweets posted by individual Twitter users as well as the information of the Twitter users are retrievable. On the contrast, through the Graph APIs of comparable social media platform Facebook, only data posted on Facebook pages but not data posted on individual users' personal accounts are retrievable. Therefore, we chose to use Twitter data in this study.

Media reports have been used in many research as the data source to extract cases of people with dementia who get lost in previous research [2, 6, 14]. We believe that social media data could also serve as similar research resources. As the nature of the contents of social media is different from that of the media - the contents of social media are usergenerated, whereas the contents in media reports are the result of the editors' choice. Therefore, in places where social media are widely used, there may be more descriptive data about people with dementia who went lost on the social media platform than in media reports. However, there is no such research that use social media data to investigate people with dementia who went lost. This research sought to fill the gap and to address the following research questions:

RQ1. Is the number of tweets and retweets associated with the outcome of finding?

$R Q 2$. Is the number of followers of the Twitter users who tweet and retweet associated with the outcome of finding?

RQ3. Do the media and police's efforts on social media correlate with the finding outcomes?

\section{Methods}

\subsection{Data collection}

We collected tweets through Twitter REST APIs [17] using rtweet package [30] operated on $\mathrm{R}$ version 3.3.3 [31]. An R script would search for tweets and retweets that contained "dementia" or "Alzheimer" as well as "lost" or "missing" at 8 a.m. GMT daily from April 1, 2017 to May 31, 2017. Tweets that were posted a day before were collected were included and stored in a database. The database was separated into two datasets, namely April Tweets Dataset and May Tweets Dataset. The information of the tweets obtained included: the tweet's id (called "status id"), created time (in GMT time zone), text, is retweet or not, language, (the tweet author's) user id and user screen name, (for the retweets) the original tweet's status id, the URLs the tweet contained (if any), etc.

All user ids obtained from the April Tweets Dataset were used to further retrieve the information of users who tweeted or retweeted relevant tweets on 30 April, 2017 and the information was stored in the April Users Dataset. All user ids obtained from the May Tweets Dataset were used to retrieve user information on May 31, 2017 and the information was stored in the "May Users Dataset". For the users whose information was not accessible during the above search time, we further tried to obtain their information in June 2017. The information of users obtained included: user id, name, user name, description (self-descriptions by the users themselves), user account created time, number of followers, number of following, number of status, etc.

\subsection{Case identification}


Tweets in the April Tweets Dataset were used to identify individual cases of people with dementia who went lost, and the May Tweets Dataset were used to follow-up the cases in April. The texts of original tweets (to be distinguished from retweets) written in the English language from the April Tweets Dataset were processed by a natural language processing $\mathrm{R}$ package - openNLP [32] with an aim to identify three elements -- age, name and place name. Original tweets that have all the three elements identified were examined by a researcher to determine whether the tweets were really about looking for lost persons with dementia.

The confirmed cases of dementia lost were selected as "sample cases", whose age, names and place information were further manually checked by the researcher according to the text, and the webpages linked to the tweets (if any) in case the openNLP from $\mathrm{R}$ script made the information inaccurate. The names of the sample cases and the places of getting lost were used as the keyword search throughout all original tweets to confirm the correctness of information. The researcher made the decisions and clustered the tweets by cases. Status ids of the identified and clustered original tweets were then used to look up retweets in both the April and May Tweets Datasets. Both the tweets and retweets that were disseminated to look for the same sample cases were then identified.

The researcher recorded age, gender, country where they got lost, found status (in three categories: found alive, found deceased or no information) in each sample case. Five sources were used to retrieve the above information: 1) the tweet text; 2) webpages of the original tweets on Twitter (so as to inspect whether there were update information in the comments of the tweets); 3) webpages linked from the original tweets (e.g. the URLs often linked to a news report or a police alert); 4) a new tweet containing updated information. 5) Google search for the person's name. Two researchers cross-checked the results. If the missing persons were found afterwards, the found time would be recorded as the earliest available time that could be obtained from the above mentioned five kinds of sources. The found time was then converted to GMT time zone so as to calculate the lost duration, which in this study was defined as the duration from created time of the first tweet looking for a lost person and the found time collected by above methods.

\subsection{User categorization}

To categorize the users who tweeted and retweeted relevant information into three types, namely, media, police and other, a method combining keyword search and manual coding was used. If twitter user accounts with the media related descriptions includes news, media, TV, radio, fm, post, daily and weekly, a researcher would conduct manual coding to confirm whether the account is from media. Likewise, if descriptions with police, sheriff, public safety, or police station appeared in a user's account, twitter account was labelled as from a police or a police station. Twitter users who posted the original post with number of followers were above 5,000 were also check for the potential identity account to our definitions. In this study, personal accounts of journalists or police officers were categorized into "media" or "police" type.

\subsection{Data analysis}

Descriptive analyses were used to present the demographics of the cases, the outcomes of finding as well as the usage of the Twitter. For each sample case, the sum of number of tweets and retweets; the sum of number of followers of Twitter users who tweets and retweeted; and the sum of the number of media and police who tweet and retweet were calculated. T-test and Chi-square test were conducted between the above summation and the outcome of the finding (found or not found). Cases whose found status were "found alive" or "found deceased" were deemed as found whereas "no information" was deemed as not found. Networks of tweets and retweets of each case were plotted using $\mathrm{R}$ package igraph [33] and plotrix [34] to explore potential patterns. All statistical analysis was conducted by IBM SPSS Statistics 21.

\section{Results}

A total of 9,973 and 39,155 tweets were collected in April and May 2017 respectively, in which 9,926 and 39,109 respectively were classified by Twitter API as written in the English language and used for further analyses. The number of original tweets in the two months were 2,267 and 3,542 respectively. Among the original tweets collected in April, 2017, 891 of them had at least one of the three elements age, name or place name identified by the openNLP R script, and 12 out of the 891 tweets had all the three elements identified. The 12 tweets were all tweets looking for lost people with dementia, and they referred to 12 different missing people. Ten of them were selected as the sample cases, and two were excluded due to the lost time of the persons was before our study time. Using the age, name and place 
name information of the ten sample cases, a total of 34 original tweets were identified from the April and May tweet datasets. Furthermore, by using the status id of the original tweets, 1273 retweets were identified from the two months' tweets datasets. The total 1307 original tweets and retweets were posted by 1284 unique users, among them 11 users' information was not in the tweet databases. Multiple attempts to retrieve the 11 users' information were conducted in June 2017, but only six of them were obtained. For the five other users whose information could not get, their numbers of followers were assumed as zero and user type assumed as "other" in the analysis.

Seven of the ten cases with dementia who went missing were male. The age of the missing persons ranged from 62 to 90 . The ten cases happened in two countries, eight in the United States and two in the United Kingdom. Eight cases had been found, the two others' whereabouts could not be traced. The demographics of the cases, the outcome of the finding, and the key information of relevant tweets and retweets are listed in Table 1. Characteristics of Twitter users who tweeted and retweeted could be seen in Table 2.

The eight found cases had a mean age of 68.9. A total of 32 original tweets and 1245 retweets were disseminated to look for the eight cases, among them $27(84.4 \%)$ original tweets and $423(40 \%)$ retweets were spread before the persons were found. Those who tweeted and retweeted before the persons were found had a total of 1,917,491 followers (4,261 followers per tweet). Seven (1.6\%) were posted by police with a total of 81,116 followers and $15(3.3 \%)$ were posted by media with a total of $1,432,416$ $(74.7 \%)$ followers. The networks of tweet and retweet of four found cases are displayed in Figure 1(a).

The two cases who were not found had a mean age of 85.5. A total of 2 original tweets and 28 retweets were disseminated within 30 days after the first tweets were posted to look for them. They have a total of 29,257 followers (975.2 followers per tweet). The networks of tweet and retweet of the two not found cases are shown in Figure 1(b).

T-test showed that the average number of tweet and retweet for found and not found cases were 56.25 and 15 respectively, without significant difference ( $\mathrm{p}$ value $=0.581$ ); and the number of followers who tweeted and retweeted was 239,686.4 for found cases and 14,628.5 for not found cases, there was no significant difference $(\mathrm{p}$-value $=0.055)$.

Chi-square analysis showed that among the eight found cases, seven $(87.5 \%)$ of them had media posted tweets, there was a statistical significantly association between the media involvement and found ( $\mathrm{p}$-value = $0.016)$; six $(75 \%)$ of them are male, there was no statistically significant association between gender and outcome of finding ( $\mathrm{p}$-value $=0.490)$; four $(50 \%)$ of them had police involvement, there was no statistical significantly association between the police involvement and outcome of finding ( $\mathrm{p}$-value = 1.000).

\section{Discussions}

This study showed that there is a promising future using social media in the research of people with dementia who are being lost. Data obtained from social media have several advantages: 1) the data often contain rich information, including a description of the missing persons (such as their ages, names, other illnesses they have), under what circumstances did the persons get lost (such as lost time, lost location), and URLs linking to police alerts or media reports with more detailed information and updates (such as the found time and found location); 2) comparing to data from clinical records, surveys or interviews that are often restrained to a certain community, data extracted from social media could include cases from around the globe; 3 ) comparing to the cases from media reports that are often the "editor's choice", the user-generated data from social media are maybe more diverse, for example, a person with dementia who went lost and were found in one hour may not be reported by the media, but there is a chance that it can be traced on social media.

Some results of this research were consistent with previous research using data from media reports or police records. In terms of gender of the lost persons, this research found that there were more males than females with dementia who become lost. This is in line with the results of previous research that used newspaper articles by Rowe and Bennett [4] and Silver Alert reports by Rowe [22]. However, other research found that age and gender are not significant factors of getting lost $[15,24]$. In terms of the country where the cases happened, this research showed that the majority of the tweets looking for people with dementia who got lost were from the United States and the United Kingdom. Two explanations may be rooted in this phenomenon: 1) most Twitter users are from the United States and the United Kingdom [35] and 2) the effectiveness of the country's "Silver Alert" systems. In terms of the outcomes of finding, this research found that $80 \%$ of the cases were found, which is similar to the research of Rowe et al. on missing drivers with dementia. Their research, based on Silver Alert reports, found 
that $83.8 \%$ of the lost were found alive, $11 \%$ were found injured and $5.1 \%$ were found dead [22].

For associations between some features of social media usage and the outcomes of finding, although statistical analysis showed that the number of followers who tweeted and retweeted was not significantly associated with the outcomes of findings, the p-value was 0.055. Considering the sample size of this research was quite small, there is a big possibility that significant correlations between the two would be found when the sample size becomes larger. This can be supported by the result that there was a significant correlation between the involvement of media and the outcomes of finding. Therefore, social media users, especially the influential ones such as the media, should be encouraged to spread the information when a person with dementia become lost.

It should be noted that although our research focused on the utility of social media in looking for lost people with dementia, we do not see social media should be used as the prime tool to look for the lost persons when incidents happen. As Bowen et al. have suggested: when discovering a person with dementia is lost, the ideal plan for caregivers is to utilize all available resources, such as neighbors, relatives, or store employees in a hasty (30 minutes or less) search of the immediate area before referring to additional resources such as law enforcement [13]. We suggest social media can been used as additional resources to look for the lost persons.

We also want to point out that there needs to be a code of conduct when using social media to look for lost persons with dementia. Information posted on social media looking for the lost persons should be correct, sufficient, and most importantly, update in time as soon as the lost persons were found. Our research found that after the missing persons were found, social media users were still tweeting and retweeting relevant tweets. Figure 2 shows the dissemination of two original tweets of one case. It shows that after the lost person was found, people were still retweeting. That might be due to they did not know the lost persons were already found. Therefore, for tweet authors or informed users, a good practice should be: as soon as the missing persons were found, update the whereabouts of the persons by commenting on the original tweets and/or by posting new tweets. Social media users should be noted that some social media platforms such as Twitter retrain users from editing post. Therefore, for users who come across tweets looking for lost persons, a good practice should be: check the comments of the tweets and new tweets posted by the tweet authors as well as other sources and channels for updated information first before spreading the information, instead of simply pressing the retweet button. Should all social media users, including the ones looking for lost demented people and the ones helping to spread the information, all follow a good code of conduct, the powers of social media could be used more efficiently.

\section{Limitations and future study}

Our research is limited to the small sample size. Social media data have the merits as discussed in the discussion part. However, the sweetness also comes with bitterness. We found that the large amount of raw data makes data processing work hard and timeconsuming. In this research, we found that case identification, clustering and tracing were not easy. We applied natural language processing on tweet texts to help identify cases from the tweets. However, the results were not satisfactory. Only 891 out of the total 2,267 original tweets from the April Tweets Datasets had at least one of three elements (age, name, place name) identified. That might be due to the features of social media messages, such as the informal of language, the wide use of abbreviations and the lack of context. We also have not come out with automatic methods for case clustering and outcomes tracing. Future research can resolve the difficulties by atomizing the process. We also believe that data processing will get easier and computing time is more reasonable in the era of Big Data application. In fact, although data processing procedures may be complicated when using social media data, the data collection procedures of social media data are relative faster than that of traditional data types such as survey and interviews.

The short study period is also one limitation of this research. The sample cases all went lost in April 2017 and we did not examine seasonal effects in this research. Future study can expand the study period to investigate whether there are seasonal effects when social media are used to find people with dementia who become lost.

Secondly, our data collection has limitations as we only used "dementia" or "alzheimer" as well as "lost" or "missing" as search terms. Tweets that contain other keywords, for example, other diseases that may cause dementia such as Parkinson's diseases and other verbs such as "disappear" might also be posted to look for people with dementia who become lost and our search strategy might miss them. However, due to our aim was to collect as many relevant tweets possible and at the same time reduce the chance of collecting irrelevant tweets, considering there are rate limits for Twitter API, we settled on 
these search terms. Our data were retrieved through query searches of Twitter's REST API, also named as Twitter Search API, therefore the obtained data is focused more on relevance but not completeness [36].

Thirdly, the outcomes of finding and found time were judged through a combination of five ways as mentioned in the methods part. We did not contact the caregivers or the police for verification, therefore, there is a chance that the found status we collected were incorrect and there is a high possibility that the found time we collected were later than the actual found time. Future study should contact the caregivers or police for accurate found status and found time.

Fourthly, our methods could not ensure that all tweets talking about a same person were extracted. This research used a method that combined natural language processing, manual coding and keywords search for case clustering, however, we could not assure that all tweets looking for the persons were all extracted from the datasets. In this study, the researchers found that for some lost persons, different spelling of names were used in different tweets, the researchers were able to identify and cluster these tweets as talking about the same persons only by double-checked using place names, the lost persons' photos and their lost time. Tweets that were talking about our sample cases but did not specify the lost persons' names could not be identified and included to our study. Therefore, there is possibility that that there were other tweets talking about the cases of our study but we failed to extract the tweets.

Finally, the information of Twitter users was collected at the end of each month, therefore the number of followers of the users were not exactly the number when the users tweeted or retweeted relevant tweets. Future study should collect relative users' information immediately after the tweets are collected.

This study is our preliminary attempt to make use of social media data in the research of people with dementia who get lost. There remain numerous potentials that future studies can tap into in this research area.

\section{Conclusion}

Social media can be a new source of data in the research of dementia. Our study contributes to the understanding of how social media were used as a tool to look for people with dementia who went lost. The findings suggest that social media users, especially those with a larger group of followers such as the media, should be encouraged to spread the messages that look for missing people with dementia on social media platforms. A code of conduct should be established so that the strengths of social media are fully used instead of being wasted.

\section{References}

[1] The World Health Organizations, "Dementia", retrieved from http://www.who.int/mediacentre/factsheets/fs362/en/, May 2017.

[2] Hunt, L.A., Brown, A.E., and Gilman, I.P., "Drivers with dementia and outcomes of becoming lost while driving", American Journal of Occupational Therapy, 64(2), 2010, pp.225-232.

[3] The World Health Organization, "10 facts on dementia", retrieved from http://www.who.int/features/factfiles/dementia/en/, updated April 2017.

[4] Rowe, M. A., and Bennett, V., "A look at deaths occurring in persons with dementia lost in the community", American Journal of Alzheimer's Disease \& Other Dementias ${ }^{\circledR}, 2003$, pp.343-348.

[5] Roth, M.E., "Advances in Alzheimer's disease: A review for the family physician", The Journal of family practice, 1993, 37, pp.593-607

[6] Kirkman, A. M., "Dementia in the news: The media coverage of Alzheimer's disease", Australasian Journal on Ageing, 25(2), 2006, pp.74-79.

[7] Aguero-Torres, H. and Fratiglioni, L., "Dementia is the major cause of functional dependence in the elderly: 3-year follow-up data from a population-based study", American Journal of Public Health, 88(10), 1998, pp.1452-1456.

[8] Lewis, H., "Dementia in New Zealand: improving quality in residential care. A report to the the Disability Issues Directorate”, New Zealand Ministry of Health, 2002.

[9] McShane, R., Gedling, K., Keene, J., Fairburn, C., Jacoby, R., and Hope, T., "Getting lost in dementia: a longitudinal study of a behavioral symptom. International Psychogeriatrics", 10(03), 1998, pp. 253-260.

[10] Silverstein, N. M., Flaherty, G., and Salmons, T., "Dementia and Wandering Behavior: Concern for the Lost Elder”, Springer Publishing Company, New York, 2006.

[11] Lai, C. K., and Arthur, D. G., "Wandering behaviour in people with dementia", Journal of advanced nursing 44.2, 2003, pp.173-182.

[12] Algase, D.L., Antonakos, C.L., Beattie, E., Beel-Bates, C.A., and Yao, L., "New parameters for daytime 
wandering", Research in gerontological nursing, 2(1), 2009, pp.58-68.

[13] Bowen, M.E., McKenzie, B., Steis, M. and Rowe, M., "Prevalence of and antecedents to dementia-related missing incidents in the community", Dementia and geriatric cognitive disorders, 31(6), 2011, pp.406-412.

[14] Rowe, M.A., Vandeveer, S.S., Greenblum, C.A., List, C.N., Fernandez, R.M., Mixson, N.E. and Ahn, H.C., "Persons with dementia missing in the community: Is it wandering or something unique?" BMC geriatrics, 11(1), 2011, p.28.

[15] White E. B. and Montgomery P., "Supporting people with dementia to walkabout safely outdoors: development of a structured model of assessment", Health \& Social Care in the Community, 2016, pp. 473-484.

[16] Algase, D.L., Moore, D.H., Vandeweerd, C., and Gavin-Dreschnack, D.J., "Mapping the maze of terms and definitions in dementia-related wandering", Aging \& mental health, 11(6), 2007, pp.686-698.

[17] "Twitter Developer Documentation, REST APIs" Retrieved from https://dev.twitter.com/rest/public

[18] Hwang, J.P., Yang, C.H., Tsai, S.J., and Liu, K.M., "Behavioural disturbances in psychiatric inpatients with dementia of the Alzheimer's type in Taiwan", International Journal of Geriatric Psychiatry, 12(9), 1997, pp.902-906.

[19] Holzer, C. and Warshaw, G., "Clues to early Alzheimer dementia in the outpatient setting", Archives of Family Medicine, 9(10), 2000, p.1066.

[20] Koester, R.J. and Stooksbury, D.E., "The lost Alzheimer's and related disorders search subject: new research and perspectives", Response, 98, 1998, pp.165181.

[21] Kinney, J.M., Kart, C.S., Murdoch, L.D., and Ziemba, T.F., "Challenges in caregiving and creative solutions: using technology to facilitate caring for a relative with dementia”, Ageing International, 28(3), 2003, Fpp.295-313.

[22] Rowe, M.A., Greenblum, C.A., Boltz, M. and Galvin, J.E., "Missing drivers with dementia: Antecedents and recovery", Journal of the American Geriatrics Society, 60(11), 2012, pp.2063-2069.

[23] Ballard, C., Lowery, K., Powell, I., O'Brien, J., and James, I., "Impact of behavioral and psychological symptoms of dementia on caregivers", International Psychogeriatrics, 12(S1), 2000, pp.93-105.

[24] Kwok, T.C., Yuen, K.S., Ho, F.K., and Chan, W.M., "Getting lost in the community: a phone survey on the community - dwelling demented people in Hong Kong", International journal of geriatric psychiatry, 25(4), 2010, pp.427-432.
[25] Rowe, M.A., "People with Dementia Who Become Lost: Preventing injuries and death", AJN The American Journal of Nursing, 103(7), 2003, pp.32-39.

[26] Carr, D., Muschert, G.W., Kinney, J., Robbins, E., Petonito, G., Manning, L., and Brown, J.S., "Silver alerts and the problem of missing adults with dementia", The Gerontologist, 50(2), 2010, pp.149-157.

[27] Obar, J.A and Wildman, S.S., "Social media definition and the governance challenge: An introduction to the special issue", Telecommunications policy, 39 (9), 2015, pp.745-750.

[28] eMarketer, "Number of social media users worldwide from 2010 to 2020 (in billions)", retrieved from https://www.statista.com/statistics/278414/number-ofworldwide-social-network-users/ (accessed June 10, 2017).

[29] Twitter, "About Twitter", retrieved from https://about.twitter.com/company, June 2016.

[30] Kearney, M. W., "rtweet: Collecting Twitter Data", R package version 0.4.0, retrieved from https://cran.rproject.org/package=rtweet, 2016.

[31] R Core Team, "R: A language and environment for statistical computing. $\mathrm{R}$ Foundation for Statistical Computing", retrieved from https://www.R-project.org/ Vienna, Austria, 2017.

[32] Hornik, K., "openNLP: Apache OpenNLP Tools Interface", $\mathrm{R}$ package version $0.2-6$, retrieved from https://CRAN.R-project.org/package=openNLP, 2016.

[33] Csardi G. and Nepusz T., "The igraph software package for complex network research", InterJournal, Complex Systems, 2006, p. 1695.

[34] Lemon, J. Plotrix: a package in the red light district of R. R-News, 6(4), 2006, pp.8-12.

[35] Statista (Digital Market Outlook), “ Number of active Twitter users in leading markets as of May 2016 (in millions)", Statista, retrieved from https://www.statista.com/statistics/242606/number-ofactive-twitter-users-in-selected-countries/

[36] Twitter, "Twitter Developer Documentation-The Search API", retrieved from https://dev.twitter.com/rest/public/search 
Table 1. Baseline characteristics, tweets, followers and found time

\begin{tabular}{|c|c|c|c|c|c|c|c|c|c|c|}
\hline Case & Age & Country & Sex & $\begin{array}{l}\text { Found } \\
\text { status }\end{array}$ & Example of tweet text & $\begin{array}{l}\text { Tweet before } \\
\text { found (total } \\
\text { tweet) / Tweet } \\
\text { in } 30 \text { days }\end{array}$ & $\begin{array}{l}\text { Retweet } \\
\text { before found } \\
\text { (total tweet) / } \\
\text { Tweet in } 30 \\
\text { days }\end{array}$ & $\begin{array}{l}\text { Sum number of } \\
\text { followers of tweet and } \\
\text { retweet before found } \\
\text { (total tweet)/ Tweet in } \\
30 \text { days }\end{array}$ & $\begin{array}{l}\text { First } \\
\text { tweet } \\
\text { time } \\
\text { stamp }\end{array}$ & $\begin{array}{l}\text { Found } \\
\text { time } \\
\text { (hour) }\end{array}$ \\
\hline 1 & 71 & US & $\mathrm{m}$ & Alive & $\begin{array}{l}\text { A 71-year-old tourist with dementia is } \\
\text { missing after walking out of a Times } \\
\text { Square hotel }\end{array}$ & $3(4)$ & $21(21)$ & $616,156(616,262)$ & $\begin{array}{l}04-25 \\
18: 35: 07\end{array}$ & 14.9 \\
\hline 2 & 65 & US & $\mathrm{f}$ & Alive & $\begin{array}{l}\text { MISSING PERSON: } 65 \text { yr old woman } \\
\text { missing since } 4 \mathrm{pm} \text { wearing black dress last } \\
\text { seen on Cliff Rd. Suffers from dementia. }\end{array}$ & $6(9)$ & $271(1082)$ & $635,789(1,425,935)$ & $\begin{array}{l}04-05 \\
00: 36: 52\end{array}$ & 2.3 \\
\hline 3 & 70 & US & $\mathrm{f}$ & Alive & $\begin{array}{l}\text { Greenville County deputies searching for } \\
\text { missing woman with Alzheimer's }\end{array}$ & $10(11)$ & $8(8)$ & $412,214(415,308)$ & $\begin{array}{l}04-13 \\
10: 02: 20\end{array}$ & 5.0 \\
\hline 4 & 62 & US & $\mathrm{m}$ & Alive & $\begin{array}{l}\text { Please RT } \sim \text { X, } 62 \text { years old, missing at-risk } \\
\text { due to dementia, last seen in Rancho } \\
\text { Cordova yesterday. }\end{array}$ & $1(1)$ & $12(20)$ & $14,189(17,760)$ & $\begin{array}{l}04-18 \\
19: 46: 11\end{array}$ & 2.0 \\
\hline 5 & 68 & US & $\mathrm{m}$ & Alive & $\begin{array}{l}\text { Missing } 68 \text { yr old white male with dementia } \\
\text { X northern City near Dover on foot dark } \\
\text { jacket }\end{array}$ & $1(1)$ & $0(0)$ & $3,299(3,299)$ & $\begin{array}{l}04-06 \\
16: 03: 57\end{array}$ & 2.7 \\
\hline 6 & 79 & UK & $\mathrm{m}$ & Alive & $\begin{array}{l}\text { Please RT: have you seen X? The 'high risk' } \\
\text { \#missing pensioner has dementia, was last } \\
\text { seen near \#Bude... }\end{array}$ & $4(4)$ & $111(114)$ & $195,741(197,753)$ & $\begin{array}{l}04-23 \\
18: 49: 42\end{array}$ & 19.9 \\
\hline 7 & 63 & US & $\mathrm{m}$ & Alive & $\begin{array}{l}\text { \#SilverAlert out of \#AmherstNY - this is } \\
\mathrm{X} \text {, last seen on South Union Road-63- } \\
\text { year-old is missing, suffers... }\end{array}$ & $1(1)$ & $0(0)$ & $17,713(17,713)$ & $\begin{array}{l}04-28 \\
19: 28: 09\end{array}$ & 3.1 \\
\hline 8 & 73 & US & $\mathrm{m}$ & Alive & $\begin{array}{l}\text { Have you seen } 73 \text {-year old X of Lexington? } \\
\text { Missing since Tuesday morning. Has } \\
\text { Alzheimer's and is diabetic }\end{array}$ & $1(1)$ & $0(0)$ & $22,390(22,390)$ & $\begin{array}{l}04-25 \\
23: 32: 55\end{array}$ & 2.4 \\
\hline 9 & 81 & US & $\mathrm{f}$ & $\begin{array}{l}\text { Not } \\
\text { found }\end{array}$ & $\begin{array}{l}\text { Help>@ @olsomPolice At risk missing } 81 \\
\text { year old female X blue sweater, blue pants } \\
\text { near Willow Creek Dr. she suffers from } \\
\text { dementia }\end{array}$ & 1 & 2 & 4,059 & & \\
\hline 10 & 90 & UK & $\mathrm{m}$ & $\begin{array}{l}\text { Not } \\
\text { found }\end{array}$ & $\begin{array}{l}\text { *MISSING PERSON* X } 90 \text { yrs with } \\
\text { dementia from Cumberland Rd. He is white } \\
\& \text { slim with short wht hair. Please contact } \\
\text { with sightings!! }\end{array}$ & 1 & 26 & 25,198 & & \\
\hline
\end{tabular}




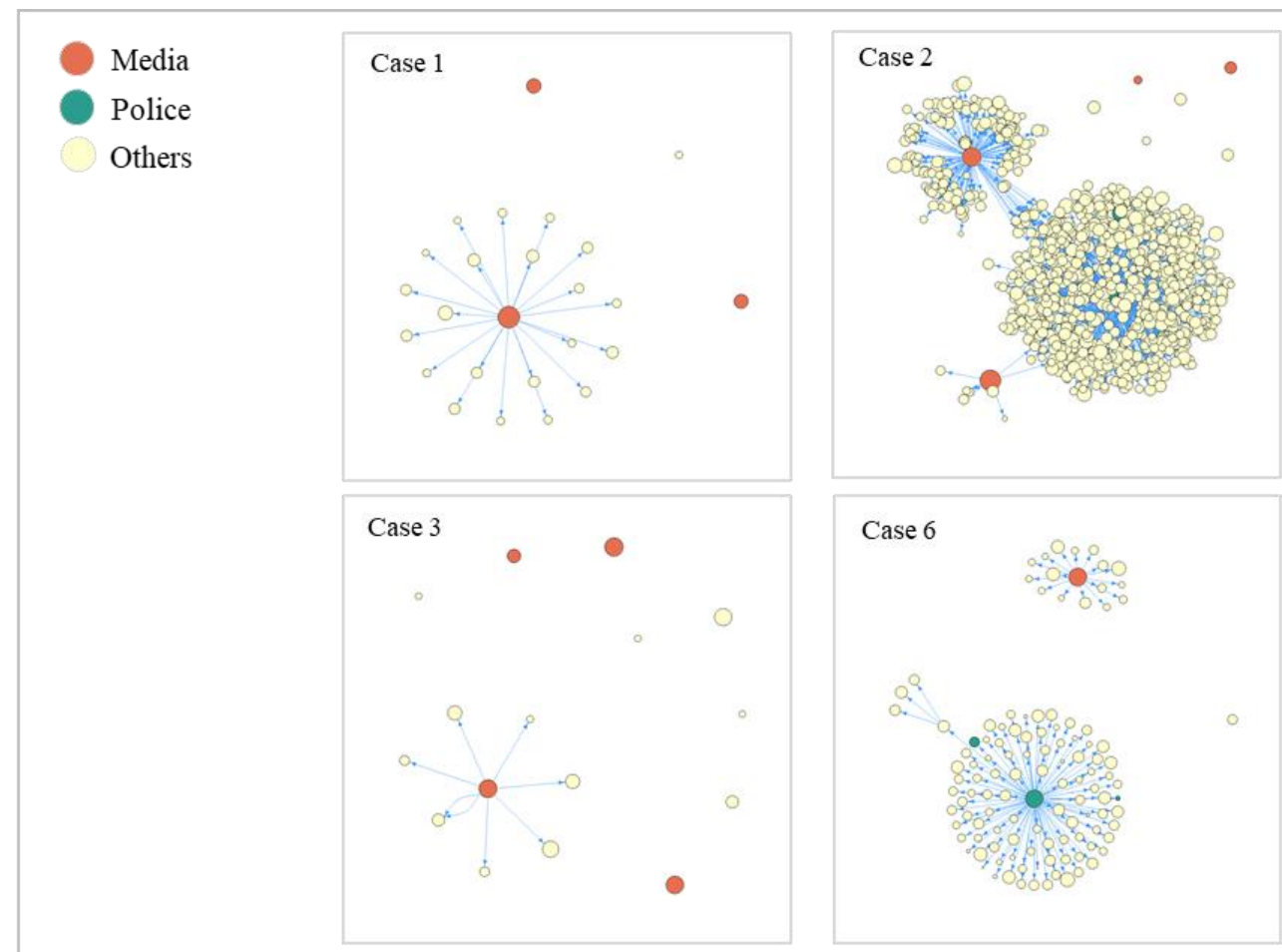

Figure 1 (a). Network of tweets and retweets of four found cases

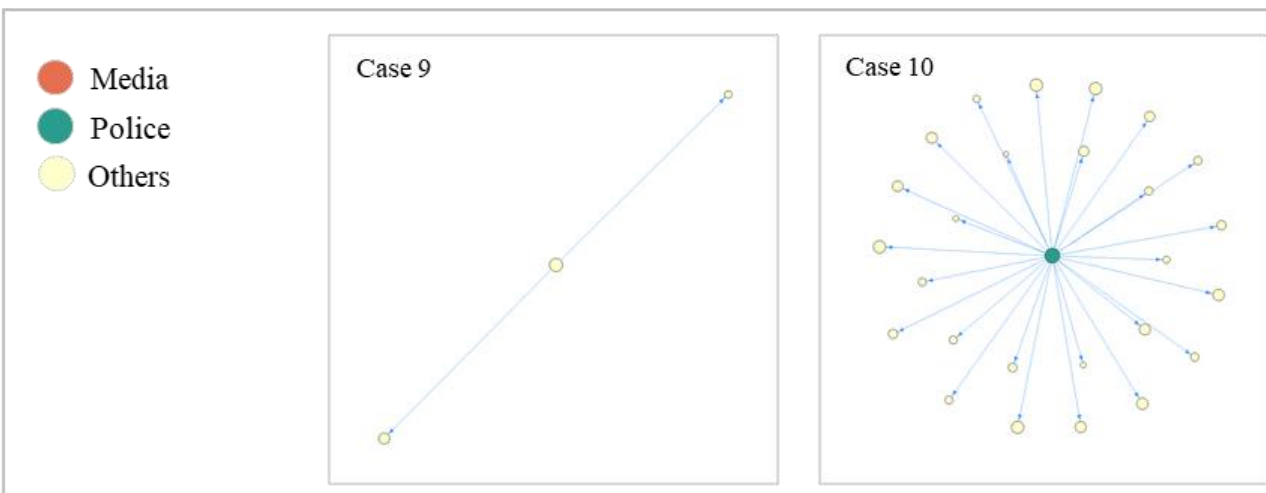

Figure 1 (b). Network of tweets and retweets of two unfound cases

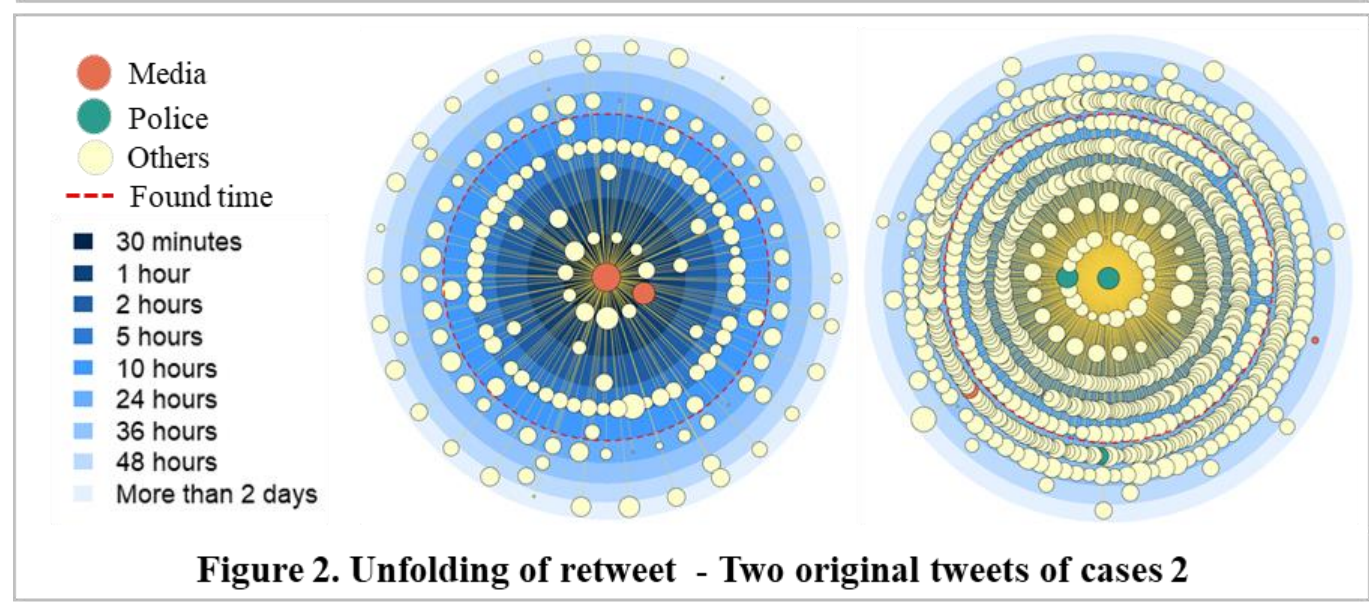

In Figure 1 and 2, size of nodes represents the log of the number of followers 